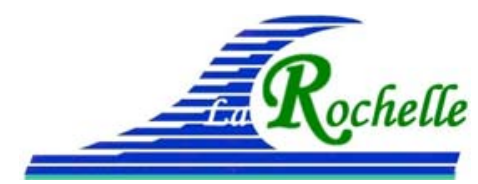

XVèmes Journées Nationales Génie Côtier - Génie Civil

La Rochelle, 29 au 31 mai 2018

DOI:10.5150/jngcgc.2018.056

(c) Editions Paralia CFL

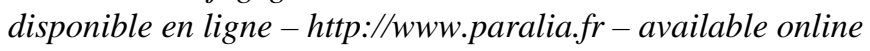

\title{
Instrumentation des structures portuaires: quelle valeur ajoutée pour la maintenance?
}

\section{Franck SCHOEFS ${ }^{1}$, Mestapha OUMOUNI ${ }^{1}$, Yann LECIEUX ${ }^{1}$, Cyril LUPI ${ }^{1}$, Virginie GAILLARD ${ }^{2}$, Michel ROCHE ${ }^{1}$, Emilio BASTISDAS-ARTEAGA ${ }^{\text {, }}$ Dominique FOLLUT ${ }^{3}$, Pascal LIJOUR ${ }^{4}$, Marc LABEGORRE 4}

\author{
1. Université de Nantes, UFR Sciences et Techniques, GeM UMR 6183 CNRS / IUML \\ FR 3473 CNRS, 2 rue de la Houssinière, BP 92208, 44322 Nantes, France. \\ Franck.schoefs@univ-nantes.fr \\ 2. ICAM, GeM UMR 6183 CNRS UMR 6143 CNRS, Nantes, France. \\ 3. KEOPS Performance, Nantes, France. \\ 4. Grand Port Maritime de Nantes St Nazaire, Nantes, France.
}

\section{Résumé :}

L'instrumentation d'ouvrages portuaires, pour leur maintenance a souvent montré son intérêt. Son coût à l'installation (entre 0,3 et $0,5 \%$ du projet) est significatif s'il n'est pas mis en regard du bénéfice. L'ouvrage TMDC5 du Grand Port Maritime de Nantes St Nazaire a été sélectionné par l'action COST TU 1402 afin de démontrer à quel niveau d'investissement le monitoring est pertinent. Ce premier article sur le sujet pose les bases et les premiers résultats du raisonnement.

\section{Mots-clés :}

Ouvrages portuaires, Quai, Instrumentation, Contrôle non destructifs, Chlorures, Corrosion.

\section{Introduction}

Les infrastructures portuaires doivent assurer leurs fonctions pendant une durée de service très longue ; il est donc essentiel de porter une attention appropriée à leur conception, mais aussi d'effectuer une maintenance adaptée afin de maintenir un niveau de service compatible avec leurs destinations et exploitations (BOERO et al., 2009a ; BOERO et al., 2009b). En raison de leur exposition à des conditions climatiques et environmentales sévères, ces infrastructures sont sensibles à des dégradations sur leur période de service, liées à la dégradation des matériaux, à l'endommagement de leurs composants, au tassement des fondations, aux risques d'affouillement et de sédimentation.

Une approche rationnelle et efficiente de la maintenance consiste aujourd'hui à optimiser les procédures de surveillance et d'entretien (regroupées au sein de la dénomination de life-cycle management - LCM). Ces principes reposent sur une démarche nécessitant la normalisation/standardisation de la surveillance, de l'évaluation de la performance résiduelle et de la prédiction de son évolution sur la base de ces 


\section{Thème 4 - Ouvrages portuaires, offshore et de plaisance}

données, de l'évaluation de nouveaux plans d'utilisation si besoin est, de la durée de service résiduelle et du coût global (exploitation, externalités) d'utilisation de l'infrastructure en conditions d'exploitation modifiées. La surveillance apparaît comme un élément clé pour une évaluation pertinente, quantitative et prédictive de la performance.

Le projet iMARECO2, lancé en 2016, vise à développer une stratégie en coût global des infrastructures maritimes par l'implémentation de systèmes de surveillance innovants et robustes afin d'optimiser leur gestion mais également de contribuer au retour d'expérience. En effet, le caractère prototype, rare et en environnement complexe des structures portuaires (zones de dégradation multiples), génère une allocation des coûts spécifique : à la conception par la méconnaissance de certains effets et l'absence de prise en compte de certaines spécificités dans les règlements, à la maintenance par la difficulté d'inspection, la complexité des pathologies et le faible retour d'expérience. Ce projet, couplant mesures in-situ et en laboratoire et méthodes d'analyse des données, a pour objectif final de contribuer à une optimisation de l'allocation des ressources intégrant le coût d'une instrumentation performante depuis la chaîne de mesure jusqu'à l'utilisation aussi bien en termes d'efficacité (robustesse / durabilité) que dans l'implantation physique sur l'infrastructure.

Cet article constitue une première phase du calcul de la valeur ajoutée de l'instrumentation pour la réduction des coûts de maintenance de poutres corrodées par la pénétration d'ions chlorures. Il s'appuie sur les travaux de l'action européenne COST TU 1402 "Quantifying the Added Value of Structural Health Monitoring".

\section{Description du quai et de son instrumentation}

\subsection{Présentation du quai}

Ce projet s'appuiera sur la réalisation du "quai EMR" commandé par le Grand Port Maritime de Nantes - Saint Nazaire et réalisé par Bouygues TPRF. Il s'agit du Terminal à marchandises diverses et conteneurs (TMDC), de $350 \mathrm{~m}$ de long et $50 \mathrm{~m}$ de large situé à Montoir dans le prolongement d'un quai existant (voir figure 1). 


\section{XVèmes Journées Nationales Génie Côtier - Génie Civil \\ La Rochelle, 29 au 31 mai 2018}
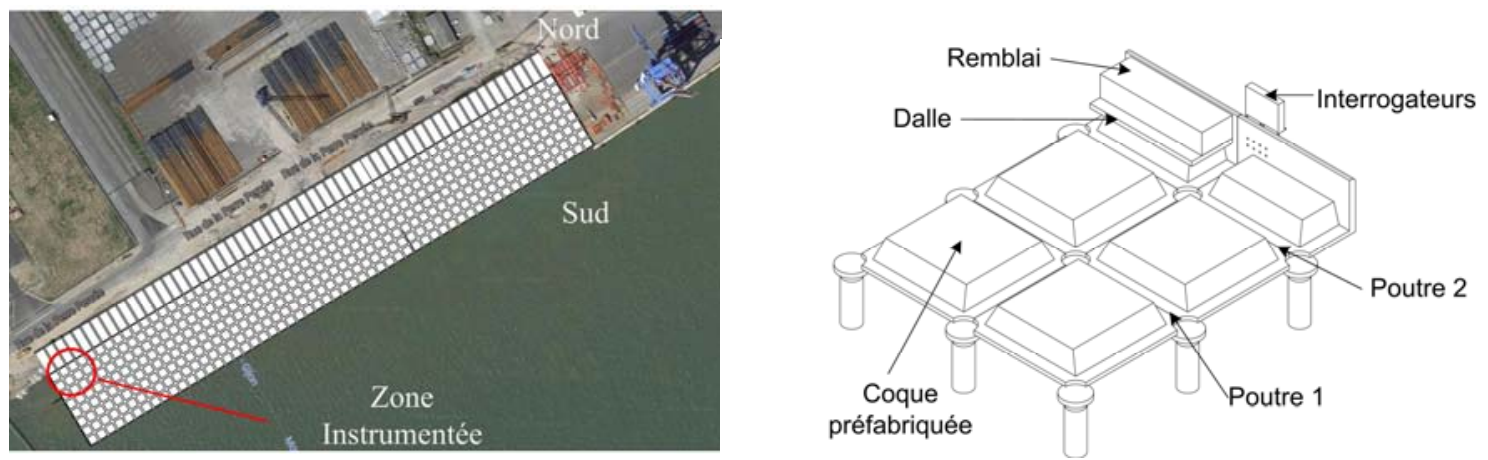

Figure 1. Ouvrage instrumenté.

L'ensemble est constitué d'une structure composée d'éléments préfabriqués en béton armé, soutenus par un maillage de 579 pieux métalliques répartis sur 10 files longitudinales et 56 files transversales. Selon les zones du quai, différentes surcharges de service sont prévues $(6,10$ ou $15 \mathrm{~T} / \mathrm{m} 2)$ ainsi que des voies de grues. Sur la structure en béton armé, une couche de remblai et un enrobé viennent habiller la plateforme. Des bollards et des défenses d'accostage viennent ensuite équiper l'ensemble.

Pour stabiliser les berges sur lesquelles s'adosse le quai, des inclusions en béton ont été réalisées et un double rideau de palplanches avec un système de tirantage a été mis en œuvre.

Les principaux chiffres du quai sont :

- 579 tubes (soit 7900 tonnes d'acier),

- $40000 \mathrm{~m} 3$ de béton (dont 17000 pour remplissage des pieux),

- 4500 tonnes d'armatures,

- 80000 heures de main d'œuvre.

\subsection{Instrumentation du quai}

Deux poutres situées en extrémités du quai sont instrumentées pour le suivi de pénétration des ions chlorures et le vieillissement associé suivant les schémas de la figure 2. Ces poutres sont repérées sur la figure 1.

Pour chaque poutre, Le choix a été fait d'associer, deux capteurs de résistivité suivant la technologie proposée par LECIEUX et al. (2015), 3 capteurs de chlorures (capteurs physico-chimiques avec 3 niveaux d'électrolytes, 3 mesures de potentiel d'armature, 3 sondes de mesure d'humidité et de température, des sondes PT 100 et une fibre continue pour une mesure simultanée de déformation et de température.

Ces capteurs sont destinés à mesurer les évolutions du béton liées au vieillissement de l'ouvrage. La résistivité électrique vise à détecter de manière précoce la pénétration des ions chlorures dans le milieu. Celle-ci dépend de la porosité du béton, de sa teneur en eau, de la concentration en chlorure de l'électrolyte ainsi que de la température du milieu. Les capteurs de résistivité sont donc utilisés en association avec les capteurs 


\section{Thème 4 - Ouvrages portuaires, offshore et de plaisance}

d'humidité et de température. Les capteurs de chlorure sont destinés à détecter le front de chlorure au moment où ce dernier atteint les armatures les plus proches de l'enrobage, avant que le processus de corrosion ne débute. Enfin, les fibres optiques ont vocation à identifier les désordres découlant de la corrosion : la fissuration du béton. Les capteurs d'humidité ont été étalonnés suivant la norme AFNOR (1999). Les capteurs de résistivité ont été étalonnés dans un milieu modèle (ici de l'eau de résistivité connue) de même volume que le béton suivant la procédure proposée par LECIEUX et al. (2015) en prenant en compte les ferraillages réels (LECIEUX et al., 2017). Ces résultats de mesure sont également utilisés pour valider le modèle numérique nécessaire à l'interprétation des mesures effectuées in situ.

La poutre 1 est également équipée de 15 extensomètres à fibre optiques (voir figure 4). Ils sont positionnés suivant le plan de symétrie de la poutre à différentes hauteurs de la fibre neutre. Ces mesures de déformations doivent permettre de comprendre le fonctionnement réel de la poutre. Les extensomètres sont associés à des réseaux de Bragg libres de déformations pour mesurer la température ainsi qu'à des fibres optiques utilisées pour la mesure simultanée de déformation et de température. L'ensemble de cette chaine est étalonnée en laboratoire en procédant à des cycles de chauffage et de refroidissement dans une enceinte thermique.
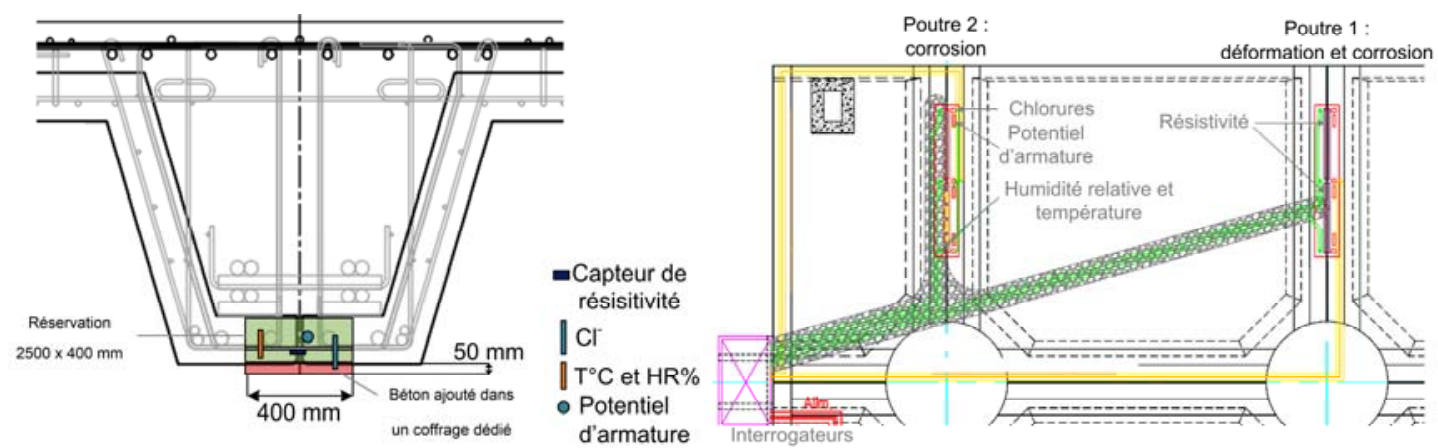

Figure 2. Positionnement des capteurs pour le suivi de pénétration des chlorures.

\section{Modélisation de la dégradation, de la mesure et calcul des coûts}

On simule la dégradation par des sauts modélisés par un processus Gamma dont les paramètres sont conformes à (EL HAJJ et al., 2017). Ici la réparation est supposée avoir les mêmes caractéristiques que le béton initial afin de limiter le nombre de paramètres d'études. C'est pourquoi, en figure 3-gauche, on présente des trajectectoires aléatoires de même nature mais différentes. On compare l'inspection par Contrôle Non Destructif (CND) réalisée périodiquement toutes les T années (10 ans en figure 3-gauche) et par Instrumentation (Structural Health Monitoring, SHM) en figure 3-droire avec un pas de mesure d'un an, suffisant pour capturer la pénétration lente des ions chlorures dans le béton. 


\section{XVèmes Journées Nationales Génie Côtier - Génie Civil \\ La Rochelle, 29 au 31 mai 2018}

Dans les deux cas, une erreur de mesure dépendant de l'état est appliquée pour représenter la réalité de la mesure. Cette erreur pourrait en effet conduire à des mauvaises décisions et donc à des surcoûts. Une maintenance préventive (PM) par remplacement du béton d'enrobage est à envisager lorsque que la concentration en chlorures atteint 0,20 ou $0,35 \%$ par $\mathrm{kg}$ de ciment au niveau des aciers et une maintenance curative $(\mathrm{CM})$ par remplacement en plus des armatures est suggérée lorsque la concentration atteint $0,45 \%$.

\subsection{Calcul de l'espérance de coûts}

On calcul le coût annuel à partir du coût total d'une stratégie selon l'équation (1).

$C(t)=c_{i} N_{i}+c_{p} N_{p}+c_{c} N_{c}$

Où $N_{i}$ est le nombre d'inspections, $N_{p}$ le nombre de réparations préventives et $N_{c}$ le nombre de réparations curatives avant la date $t$. On calcule ensuite l'espérance du coût pour chaque stratégie à partir de toutes trajectoires simulées. Les coûts unitaires par mètre linéaire sont donnés dans le tableau 1 selon BASTIDAS-ARTEAGA et SCHOEFS (2015).

Tableau 1. Synthèse des coûts en euros.

\begin{tabular}{ll}
\hline Type & Coûts /MI \\
\hline Maintenance préventive & 263,2 \\
Maintenance curative & $2000+353,4$ \\
Inspection CND & 25 \\
Instrumentation SHM & $9,5 /$ an de post-traitement +25 à installation \\
\hline
\end{tabular}

\section{Résultats}

On présente en figures 4 les résultats des coûts moyens par $\mathrm{ml}$ et par an selon différentes stratégies (intervalle de temps $\mathrm{T}$ entre inspection), pour un seuil de maintenance préventif fixé à 0,20 (droite) et $0,35 \%$ (gauche). Les courbes SHM ne dépendent pas de $\mathrm{T}$ et sont les droites horizontales. Les résultats sont présentés dans le tableau 2. Le cas bruité SHM est donné pour information car dans ce cas le bruit peut être filtré (structure stochastique différente de celui du signal: figure 3). On ne commentera pas les courbes "actual state CND" puisqu'elles correspondent à une détection parfaite de l'état qui n'est pas possible compte tenu des erreurs de mesure importantes (BONNET et al., 2017).

\subsection{Modélisation de la dégradation et de la mesure}

On modélise la pénétration des chlorures dans le béton par des sauts annuels représentés par des processus Gamma (EL HAJJ et al., 2017). On présente en figure 3 l'évolution des chlorures au droit de l'armature. 
Thème 4 - Ouvrages portuaires, offshore et de plaisance
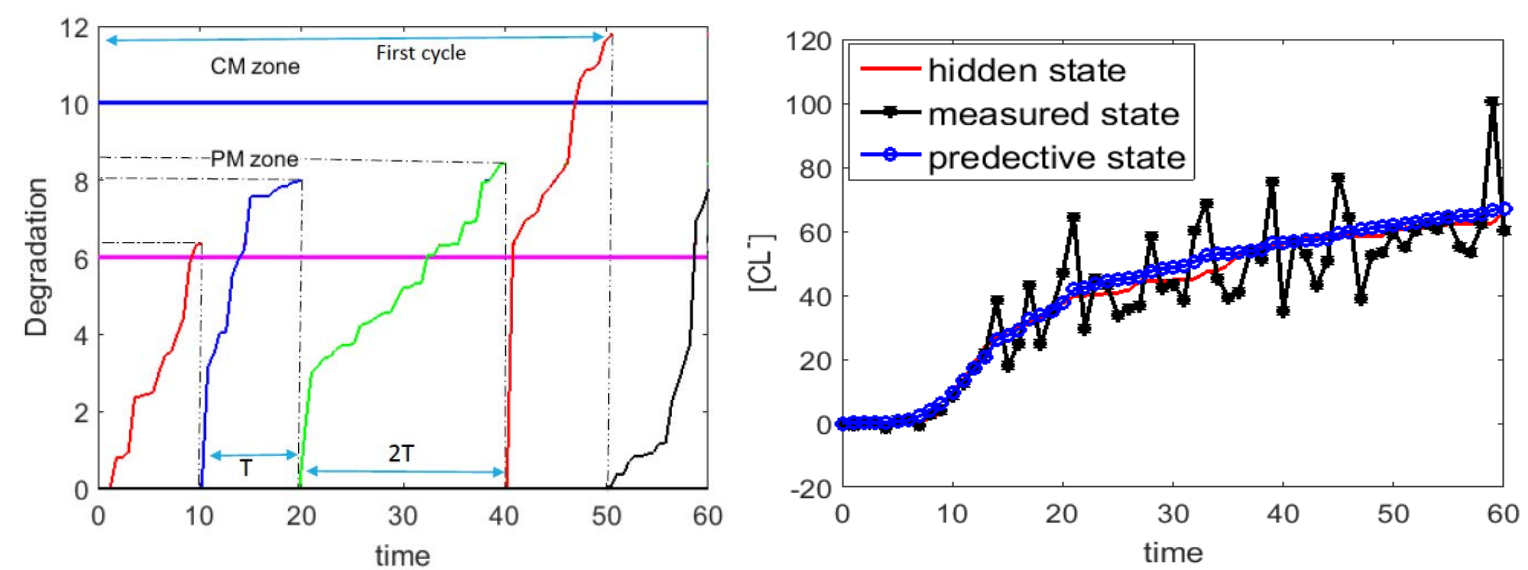

Figure 3. Simulation d'une trajectoire de chlorures (en $\left.\times 10 \mathrm{~kg} / \mathrm{m}^{3}\right)$ stratégie d'inspection tous les 10 ans (gauche). Droite : signal SHM traité en x100 de \%).
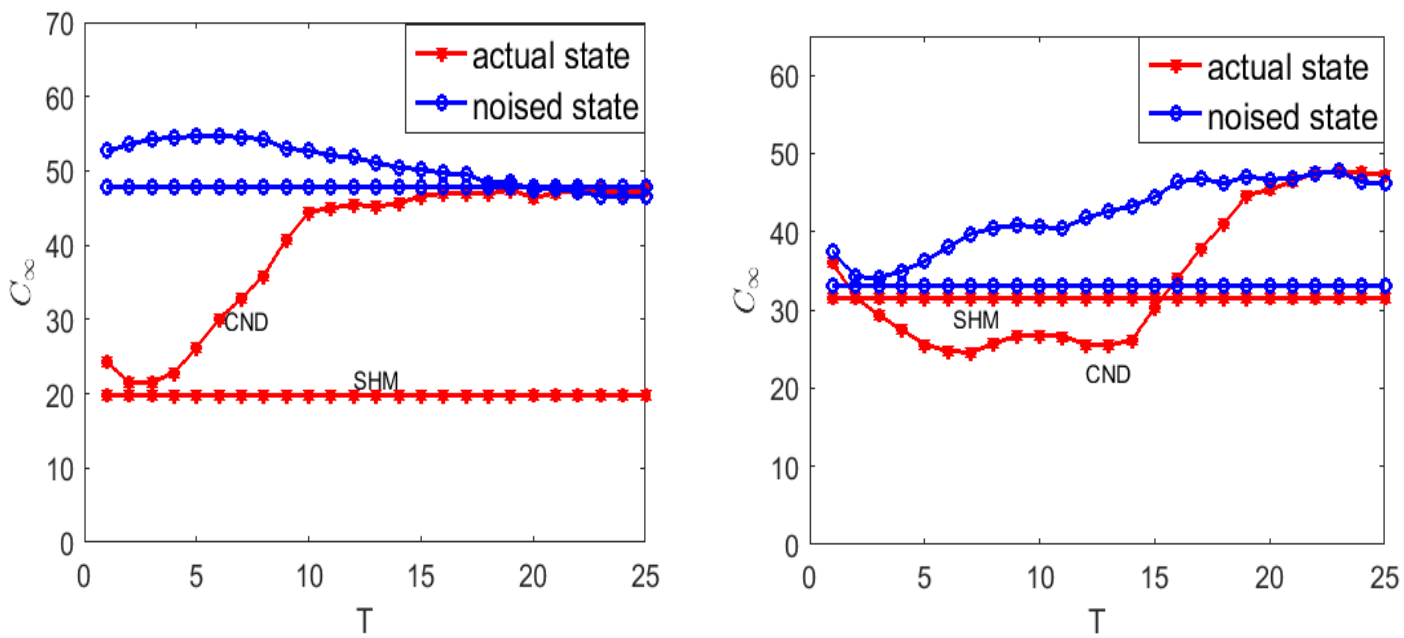

Figure 4. Coûts annuels des stratégies pour un seuil de 0,35\% (gauche) et 0,20\% (droite). En rouge, avec le processus non bruité et en bleu le processus bruité.

Tableau 2. Optimum des stratégies CND (T : période (an), CND, C : coût annuel $/ \mathrm{ml}$ ).

\begin{tabular}{lll}
\hline & Seuil $\mathbf{P M}$ à 0,35\% & Seuil $\mathbf{P M}$ à 0,20\% \\
\hline Bruité & $C N D: T=24, C=46,5$ & $C N D: T=3, C=34,1$ \\
Non bruité & $S H M: C=47,9$ & $S H M: C=33,1$ \\
& $C N D: T=20 u 3, C=21,5$ & $C N D: T=7, C=24,6$ \\
& $S H M: C=19,8$ & $S H M: C=31,5$ \\
\hline
\end{tabular}

\section{Discussion et conclusions}

On remarque qu'en l'absence de bruit de mesure les coûts sont plus faibles. Un seuil trop éloigné de la maintenance curative (cas 0,20\%) amène à réaliser une maintenance 


\section{XVèmes Journées Nationales Génie Côtier - Génie Civil La Rochelle, 29 au 31 mai 2018}

préventive trop précoce et augmente les coûts. Il est à noter qu'avec un seuil de $0,35 \%$ (qui pourra être optimisé), le CND présente des coûts nettement plus élevés ; pourtant la durée en situation de corrosion n'entraîne pas ici de pénalité ce qui devrait être le cas avec $\mathrm{T}=24$ ans. Ce critère sera ajouté dans des travaux futurs.

Cet article a permis, pour la première fois, de disposer d'une méthode de calcul de la valeur ajoutée du SHM, qui sera complétée notamment en calculant le coût de remplacement du SHM, de durée de vie limitée.

\section{Références bibliographiques}

AFNOR, (1999). NormeNFX15-119, AFNOR.

BASTIDAS-ARTEAGA E., SCHOEFS F. (2015). Sustainable Maintenance and Repair of RC Coastal Structures, Proceedings of the Institution of Civil Engineers - Maritime Engineering Journal, Vol. 168(4), pp 162-173. https://doi.org/10.1680/jmaen.14.00018

BONNET S., SCHOEFS F., SALTA M. (2017). Sources of uncertainties for total chloride profile measurements in concrete: quantization and impact on probability assessment of corrosion initiation. European Journal of Environmental and Civil Engineering, pp 1-16. https://doi.org/10.1080/19648189.2017.1375997

BOERO J., SCHOEFS F., CAPRA B., ROUXEL N. (2009). Technical management of French harbour structures - Part 1: Description of built assets. Revue Paralia, Vol. 2, pp 6.1-6.11. https://doi.org/10.5150/revue-paralia.2009.006

BOERO J., SCHOEFS F., CAPRA B., ROUXEL N. (2009). Technical management of French harbour structures - Part 2: Current practices, needs - Experience feedback of owners. Revue Paralia, Vol. 2, pp 6.13-6.24. https://doi.org/10.5150/revue-paralia.2009.007

EL HAJJ B., SCHOEFS F., CASTANIER B., YEUNG T. (2017). A condition-based deterioration model for the stochastic dependency of corrosion rate and crack propagation in corroded concrete structure, Computer Aided Civil and Infrastructure Engineering, Vol. 32, pp 18-33. https://doi.org/10.1111/mice.12208

LECIEUX Y., SCHOEFS F., BONNET S., LECIEUX T., LOPES S. P. (2015). Quantification and uncertainty analysis of a structural monitoring device : detection of chloride in concrete using DC electrical resistivity measurement, Nondestructive Testing and Evaluation, Vol. 30(3), pp 216-232. https://doi.org/10.1080/10589759.2015.1029476 LECIEUX Y., LUPI C., GAILLARD V., GUYARD R., LEDUC D., ROZIÈRE E., PRIOU J., ROCHE M., SCHOEFS F. (2017). Instrumentation d'un quai portuaire pour le suivi de vieillissement de l'ouvrage, RUGC17 (XXXVièmes Rencontres Universitaires de Génie Civil : de l'expérimentation à la modélisation en Génie Civil), Nantes (France), 22 au 24 mai 2017, 4 p. 
Thème 4 - Ouvrages portuaires, offshore et de plaisance 